\title{
Self-tuning Routine Alarm Analysis of Vibration Signals in Steam Turbine Generators
}

\author{
Jason J. A. Costello, Graduate Member, IEEE, Graeme M. West, Member, IEEE, Stephen D. J. McArthur, Senior \\ Member, IEEE, Graeme Campbell
}

\begin{abstract}
This paper presents a self-tuning framework for the diagnosis of routine alarms in steam turbine generators utilizing a combination of inductive machine learning and knowledge-based heuristics. The techniques provide a novel basis for initializing and updating time series feature extraction parameters used in the automated decision support of vibration events due to operational transients. The data-driven nature of the algorithms allows for machine-specific characteristics of individual turbines to be learned and reasoned about. The paper provides a case study illustrating the routine alarm paradigm, and the applicability of systems using self-tuning techniques. The approaches discussed throughout are presented to provide useful diagnosis tools for the reliability and maintenance analysis of steam turbine generators.
\end{abstract}

Index Terms-Nuclear power generation, condition monitoring, knowledge-based systems, time series analysis, self-tuning.

\section{LIST OF ACRONYMS}

$\begin{array}{ll}\text { AGR } & \text { Advanced Gas-cooled Reactor } \\ \text { CDF } & \text { Cumulative density function } \\ \text { ECDF } & \text { Empirical cumulative density function } \\ \text { FFT } & \text { Fast Fourier Transform } \\ \text { HP } & \text { High pressure (turbine stage) } \\ \text { ISO } & \text { International Standards Organization } \\ \text { UK } & \text { United Kingdom } \\ \text { XML } & \text { eXtensible Markup Language }\end{array}$

NOMENCLATURE

$x_{k}$

$E\left(x_{k}\right)$

$l l\left(x_{k}\right)$

$l t\left(x_{k}\right)$

$\operatorname{mo}\left(x_{k}\right)$

$u l\left(x_{k}\right)$

$u t\left(x_{k}\right)$

$\zeta(k)$

$f_{h}(x)$

$h$

$K_{h}$

$n$

$\alpha(t)$

$|S|$

\author{
$S_{\text {Lead }}$ \\ $S_{\text {Tail }}$ \\ $t_{S}$ \\ $t_{T r}$ \\ Routine step lead stability \\ Routine step tail stability \\ Routine step minimum period \\ Routine trend minimum period
}

\section{INTRODUCTION}

$\mathbf{T}$ HE ongoing monitoring and analysis of rotating machinery condition within the energy generation context plays a notable role in its safe, effective operation [1], [2]. This role is particularly true with regards to the nuclear industry, where substantial efforts are made through the regulation process to maintain accountability and safe practice in the utilization of key plant components. Measurement and interpretation of vibration signal levels is an industrially accepted health assessment technique, providing a versatile, useful machine view of rotating assets. Indeed, vibration data analysis has a noted history in research, with techniques in intelligent fault classification [3], [4], automated diagnosis [5], [6], and prognostics [7].

Alarm-driven strategies are commonly adopted by engineers to automatically identify anomalous behaviors in rotating plant assets. Such approaches to condition monitoring provide a very useful system view from a reliability and maintenance perspective, reducing the volume of low level data analysis required by experts. Limits of acceptable levels of vibration can be defined, which then trigger notifications in the event of a breach of a threshold. Engineers then use these notifications to make decisions regarding the ongoing operation of the rotating plant item in question.

However, transient conditions experienced during power plant operation corresponding to events such as outages, low power operation, and maintenance regimes, can introduce changes to base vibration levels. Though unacceptable under steady state operating conditions, these deviations are acceptable under these new changing machine states. As a result, large volumes of alarm notifications are generated, despite having a clear causal relationship to explicable changes in plant operation. Commonly referred to by engineers as routine alarms, there remains a strong requirement to identify, analyze, and document these alarms despite their benign nature with regards to the health of the machine. This is particularly true in the nuclear industry in the UK, where it is a regulatory requirement to assess every alarm generated by the installed monitoring systems on the rotating plant.

The research presented here builds on work by Todd et

al [8], who developed a prototype rule-based system for
J. J. A. Costello, G.M. West and S.D.J McArthur are with the Institute for Energy and Environment, Department of Electronic \& Electrical Engineering, University Of Strathclyde, 204 George Street, Glasgow, G1 1XW, UK email: jjacostello@eee.strath.ac.uk. G. Campbell is with EDF Energy UK, GSO Business Park, East Kilbride, Glasgow

Manuscript received January 1, 2012 
diagnosing routine alarms identified on steam turbines operated in the UK-based Advanced Gas-cooled Reactor (AGR) nuclear power plants. A limitation of this prototype system is the requirement to manually tune channel profiles for each observable for each machine, in order to transform time series machine observables to the symbolic representation required. This issue was addressed by the authors in an exploratory study of self-tuning techniques [9], using machine learning techniques to initialize channel profiles from training data.

This paper expands on this previous work through the development of a self-tuning framework for the automated diagnosis of routine alarms incident on steam turbine generators. Utilizing a combination of inductive machine learning, knowledge-based heuristics, and statistical inference, the techniques have the ability to infer envelopes of normal operation from machine training data, and provide decision support on future routine alarms. This process allows for the creation of machine-specific models of operation, providing accurate alarm analysis across multiple turbines. The automatic delivery of decision support tailored to each machine is proposed to provide an excellent tool in the general reliability analysis of the turbine generator family of assets in the energy generation context.

The paper opens by introducing a routine alarm diagnosis knowledge base, and a time series feature extraction module. The following section then presents the self-tuning framework used to optimize system parameters to previously unseen examples of turbine generator operation, allowing for diagnosis of routine alarms to be made successfully on different machine instances without the requirement for lengthy manual initialization. A case study is then presented, illustrating the tuning functionality applied to a generator load transient event on a nuclear power plant steam turbine. Finally, the paper is concluded with a discussion of the next stages of research, and the future potential for self-tuning systems in vibration analysis of rotating machinery.

\section{CONTEXT}

\section{A. Related work}

Success in the application of intelligent techniques to automated analysis, decision support, and diagnosis in asset management has seen the adoption of such systems to the monitoring of numerous rotating machines [10]. Broadly, intelligent techniques in application to condition monitoring can be considered to be either knowledge-based, or datadriven.

Knowledge-based systems utilize elicited expertise from engineers and relevant documentation to create rule bases, from which automatic diagnoses regarding operation can be made. Systems reliant on rules and heuristics have a distinguished history of application in the condition monitoring of a wide variety of engineering scenarios: turbine generators [5], [6], power transformers [11], [12], and power system fault analysis [13]. The success in application of such systems reflects the advantages of knowledge-driven approaches, having the ability to provide clear conclusions and justification behind any decision support provided.
Data-driven techniques take an inductive approach to inference, utilizing data available from current and historical operation to reason about a variety of system problems. Machine learning and data mining algorithms are utilized to benchmark or classify particular modes or features of operation, from which reasoning can be made. The construction of fault classifiers from observed behavior is particularly suited to this approach, where volumes of data are high but the physics-based knowledge of the system is lacking. A variety of techniques have been adapted to the problem of condition monitoring (specifically anomaly detection and fault classification), including neural approaches [14], parametric and non-parametric [15] machine learning, and algorithms rooted in statistical inference methods [16], [17].

A large proportion of the research in the condition monitoring of assets from an intelligent systems perspective remains in the areas of fault classification and isolation. Such techniques continue to be investigated and applied to aircraft engines [18], wind turbines [19], and gas turbines [20], to aid in the ongoing preventative maintenance regimes required in these disciplines. These classifiers are often utilized post-alarm to diagnose incipient machine faults, and provide validation to manual engineering scrutiny. Despite this approach, the automatic classification of routine instances, and the potential information to be taken from their incidence, remains unexplored with regards to turbine generators.

\section{B. Knowledge-based system development}

Routine alarm analysis has been tackled through the development of a knowledge-based system for use on vibration signals of turbine generators [8]. Through formal knowledge engineering steps [21], the development of the knowledge base used for diagnosis was made in close collaboration with vibration and rotating machinery experts. Fig. 1 provides an overview of the system within the context of use.

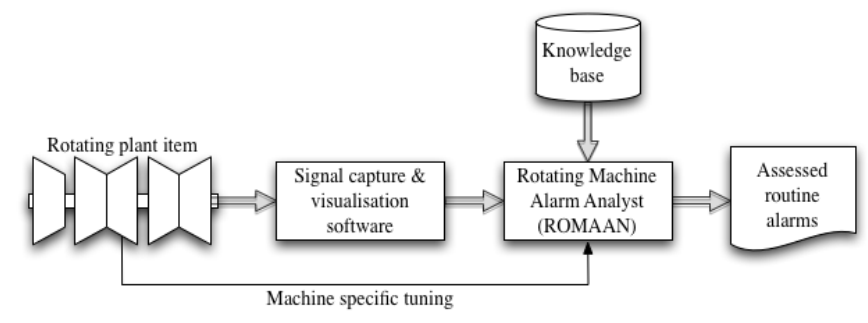

Fig. 1. Illustration of the knowledge-based system in use with a rotating plant item, delivering decision support to the engineering regarding incidences of routine alarm.

The system in [8] utilizes a crisp rule base to identify time series features within discrete alarm buffer data examples, and provides decision support regarding the causal root of the routine alarm. Typical alarm causes include operational changes, transducer noise, and loss of signal. These diagnoses are attained through the compilation of the channel profile: a collation of all identified symbols generated by the alarm buffer data on a particular observable type. Symbolic events are generated exclusively from the time series data, though 
there has been some discussion regarding the use of frequencybased Fast Fourier Transform (FFT) data to provide more diagnostic ability.

Correlation between multiple events across observables will chain together to eventually fire a corresponding diagnostic alarm causation rule. Fig. 2 gives an example showing the pre-requisites in diagnosing a generator load step change as the cause of a $1 X$ component magnitude observable change. This rule illustrates the highest level of abstraction for the rule base in diagnosing an alarm fired due to a load step change. Numerous sub-rules are required to be fired from the symbols generated in the channel profile to identify each of these features.

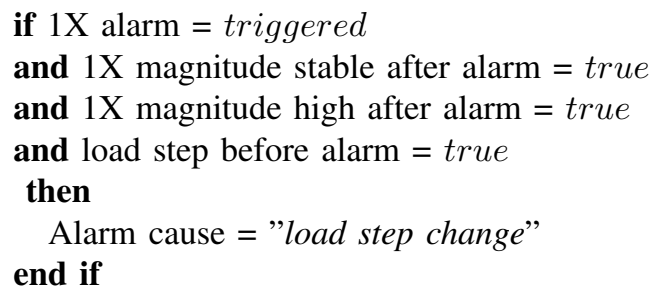

Fig. 2. Example production rule utilized to determine the cause of a routine alarm incidence following the compilation of the channel profile.

A major advantage of utilizing such a knowledge-driven, crisp approach is that of validation. The final conclusion of the system is easily communicable to the end-user by providing details of the rules fired to a suitable level of abstraction. Ensuring the communicability of any conclusion made by an automated system is of great importance to delivering useful decision support.

\section{Symbolic representation}

To use the knowledge base, the continuous time series data incident from each of the machine observables is required to be converted into a discrete representation. This conversion is achieved through signal-to-symbol transformation, a technique fairly common throughout knowledge-based systems dealing with continuous signals. Previous efforts in the decomposition of time series into primitives have introduced classification processes by which to automatically detect common features [22]. In accordance with this approach, the system utilizes three symbolic primitives corresponding to fundamental temporal events observed in the data. The process differs from much of the pattern recognition literature in that each of the primitives is defined parametrically. The machine learning and tuning of these primitive parameters provides the basis for a condition metric on the machine, which is discussed in later sections.

For a given machine, there is a corresponding machine profile representation (parsed and stored within the system in eXtensible Markup Language (XML)) which dictates the envelopes and symbolic parameters of normality for each of the asset components and channels. These parameters are the basis by which the system generates the primitive symbols from the continuous data, and thus provide the model of normality defined by the machine itself. For each vibration (a)

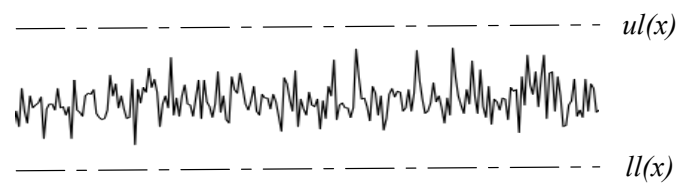

(b)

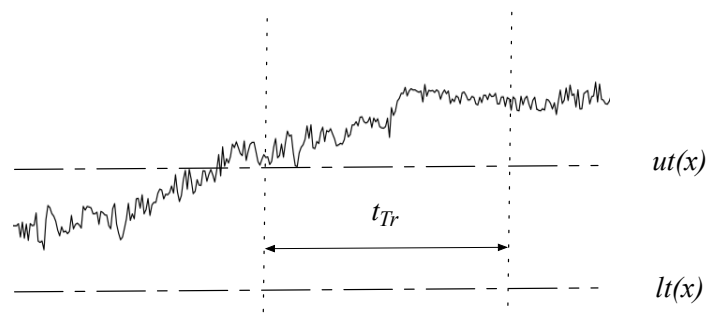

(c)

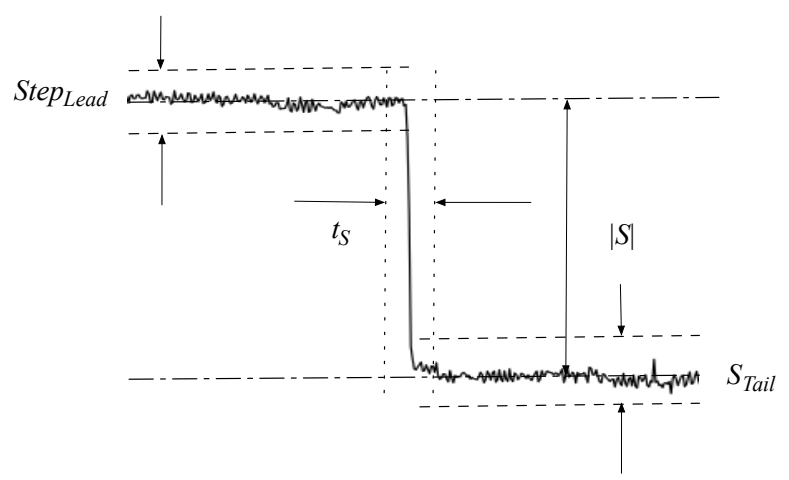

Fig. 3. Parametric representation of each of the symbolic primitives used in the signal-to-symbol transformation process: (a) level parameters $u l(x)$, and $l l(x)$; (b) trend parameters $u t(x), l t(x)$, and $t_{T r}$; and (c) step parameters $S_{\text {Lead }}, S_{\text {Tail }},|S|$, and $t_{S}$.

and operational observable utilized in the monitoring regime of the turbine, the system requires initialization of the parameters for each of the three time series primitives discussed in Table I, and illustrated in Fig. 3. Once correctly set, the feature extraction procedure (developed by Todd et al. in [8]) uses these normality parameters to identify examples of each of the three time series primitives in alarm buffer data examples.

TABLE I

DEFINITIONS OF THE PRIMITIVES USED IN GENERATING THE CHANNEL PROFILE, CORRESPONDING TO THE LATER PARAMETRIC DEFINITIONS.

\begin{tabular}{|l|l|}
\hline Primitive & Definition \\
\hline Level & $\begin{array}{l}\text { Corresponds to the threshold of allowable operation, used } \\
\text { in the classification of impulses. Defined by parameters of } \\
\text { expected average } \bar{x} \text {, and upper, lower limits }\{u l(x), l l(x)\} .\end{array}$ \\
\hline Trend & $\begin{array}{l}\text { Used in the classification of rising and falling trends } \\
\text { in the data. Defined by parameters of trend tolerances } \\
\{u t(x), l t(x)\}, \text { and minimum trend period } t_{T r} .\end{array}$ \\
\hline Step & $\begin{array}{l}\text { Used in the classification of rising and falling step changes } \\
\text { in the data. Defined by parameters of lead, tail tolerances } \\
\left\{S_{L e a d}, S_{T a i l}\right\}, \text { maximum step period } t_{S}, \text { and minimum } \\
\text { step magnitude }|S| .\end{array}$ \\
\hline
\end{tabular}




\section{SELF-TUNING APPROACH}

The procedure in the developed system has been to initialize the time series primitive parameters manually, through experience, trial and error, and corresponding to engineering standards. While this approach has proved adequate as a proof of function through case studies, the continual use in an engineering environment necessitates this lengthy process to be repeated by the end-user to account for the characteristics of multiple machines. This cost prompted the investigation of adaptive capabilities [9] to allow for the initialization process to be automated through use of machine learning techniques. This motivation to create a system with the ability to automatically identify and account for behavior in a datadriven representation of normality has driven the creation of a self-tuning framework.

\section{A. Learning parameters}

In tuning a machine profile with nominal data from a previously unseen example, we seek to automatically infer the best representation of normality for the plant item or component. The profile is defined primarily by the creation of envelopes of operation. Let $\alpha$ represent a machine or component profile with $k$ total observables:

$$
\alpha_{k}=\left[\begin{array}{ccc}
m o\left(x_{1}\right) & \ldots & m o\left(x_{k}\right) \\
l t\left(x_{1}\right) & \ldots & l t\left(x_{k}\right) \\
l l\left(x_{1}\right) & \ldots & l l\left(x_{k}\right) \\
u l\left(x_{1}\right) & \ldots & u l\left(x_{k}\right) \\
u t\left(x_{1}\right) & \ldots & u t\left(x_{k}\right) \\
t_{T r}\left(x_{1}\right) & \ldots & t_{T r}\left(x_{k}\right) \\
|S|\left(x_{1}\right) & \ldots & |S|\left(x_{k}\right) \\
t_{S}\left(x_{1}\right) & \ldots & t_{S}\left(x_{k}\right) \\
S_{\text {Lead }}\left(x_{1}\right) & \ldots & S_{\text {Lead }}\left(x_{k}\right) \\
S_{\text {Tail }}\left(x_{1}\right) & \ldots & S_{\text {Tail }}\left(x_{k}\right)
\end{array}\right]
$$

These values provide the symbolic thresholds from which the knowledge base denotes changes in behavior as either routine or anomalous. A single observable view for the $k^{t h}$ observable can therefore be considered as the vector $\zeta(x)$, where $\alpha_{k}=[\zeta(1), \zeta(2), \cdots, \zeta(k)]^{T}$, a collection of all parametric views of observables for a given machine.

These parameters can be considered in two separate sets: those defined as envelopes of steady state data, and those defined on an event-by-event basis. Values corresponding to the level and trend definition boundaries fall into the envelope category, while the parameters such as step magnitude and trend period need to be analyzed in an instance-based manner.

The machine profile approach negates inter-observable correlation in favor of a non-application dependent framework. In some domains, however, the implicit correlations between parameters may be of use to more advanced diagnostic techniques. This correlation should be considered when applying the framework beyond the scope of simple routine alarm identification.

1) Envelope-based parameters: To infer the normal bounds of operation for a new, previously unseen observable or collection of observables, the envelopes of operation are defined statistically from system behavior labelled as normal. The

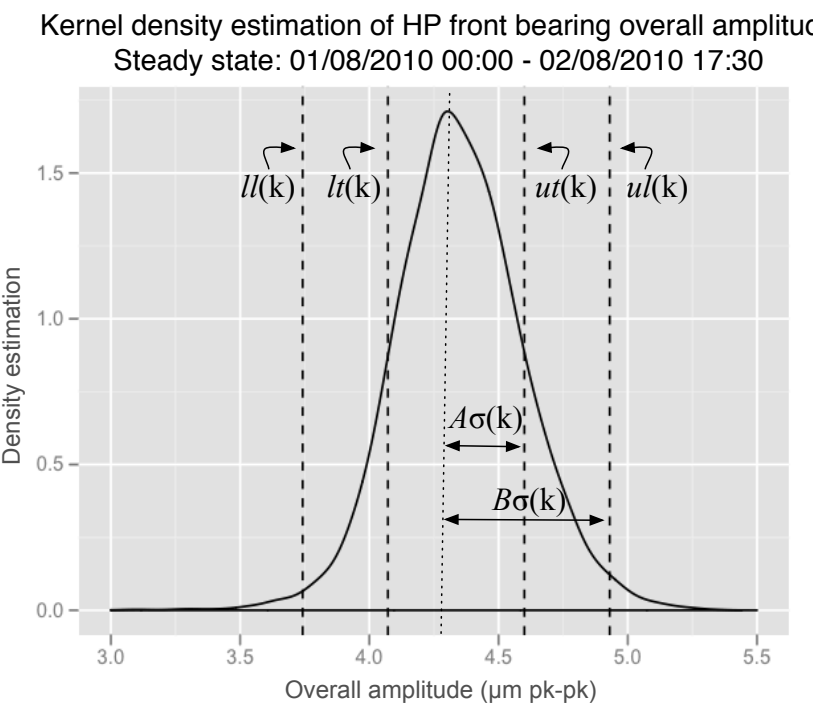

Fig. 4. Example probability density function, computed by following the process of kernel density estimation.

selection of these data is taken from the operational history of the machine. For example, an ideal candidate for tuning data would be a sample of steady state data sufficiently after the previous outage, maintenance cycle, or operational change of the machine, with consideration of any lasting transients. This period is reasoned to best represent the machine in its normal state. Kernel density estimation (also referred to as 'Parzen windows') provides a useful observable view to both reason about the system behavior and initialize the parametric envelope. This technique creates a visualization of the probability distribution function by representing each observable instance with a kernel function. Summation of these kernels provides the final behavior distribution.

The function $f_{h}(x)$, defined as

$$
\begin{gathered}
f_{h}(x)=\frac{1}{n} \sum_{i=1}^{n} K_{h}\left(\frac{E(x)-x_{i}}{h}\right) \\
\text { where } K_{h}(u)=\frac{1}{\sqrt{2 \pi}} \exp \left(-\frac{u^{2}}{2}\right)
\end{gathered}
$$

provides the estimation function. Fig. 4 shows an example probability density function derived through the kernel estimation technique, with appended envelope parameter settings (the example given is of the front transducer of a high pressure (HP) bearing).

The values for the envelope boundaries, which define the normal region of observable behavior, are determined as factors of the standard deviation $\sigma$ of the data, where

$$
\begin{aligned}
l t(k) & =m o(x(k))-A \sigma(k) \\
l l(k) & =m o(x(k))-B \sigma(k) \\
u t(k) & =m o(x(k))+A \sigma(k) \\
u l(k) & =m o(x(k))+B \sigma(k)
\end{aligned}
$$


Kernel density estimation of HP front bearing overall amplitude Step transient: 22/06/2006 00:05 - 20/07/2010 09:55

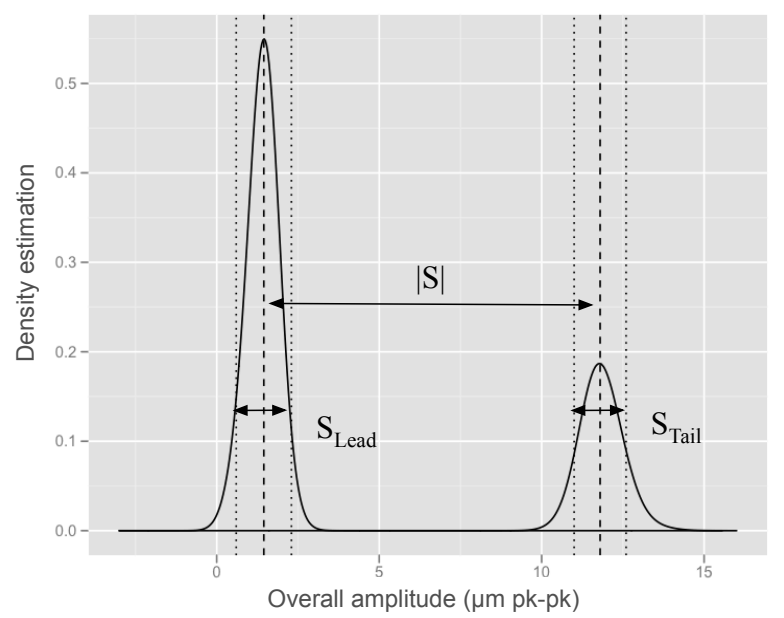

Fig. 6. Kernel density estimation for step transient example. Appended are the parameters required by the machine profile to define routine step changes.

These expressions provide the two major envelopes of operation from which anomalies can be defined symbolically, with the tolerance envelope corresponding to trend detection, and the limit envelope corresponding to the absolute boundaries for the behavior. The $A$ and $B$ parameters represent a domainspecific free parameter that requires optimization to denote the correct sensitivity of deviation detection for both envelopes.

This distribution is used primarily as visualization of nominal data prior to tuning, but also as a probabilistic measure of the observable behavior. The $m o(x)$ value of each $\zeta(k)$ corresponds to the dominant mode of the probability distribution at the most peaked observable value. In the event of varying and unstable observables, the detection of multimodal distributions discriminates against unsuitable data being used for tuning.

2) Instance-based parameters: The remaining parameters within the machine profile (step transient parameters, and minimum trend period) are required to be initialized and tuned through an instance-based methodology. These instances are of a routine nature. That is, the system seeks to infer a definition for a step change or trend period corresponding to an understood, identifiable operational change. Discrimination from transients due to system damage or change is important, as the machine profile is required to define the machine view of normal operation.

The initialization of instance-based parameters is accomplished at this stage by manual selection of training data of historical step and trend examples. From these instances, the parametric values of each primitive can be defined through similar means as in the envelope-based parameters.

Fig. 6 provides the kernel estimation visualization of an example transient, along with the parameters of interest required by the machine profile. The step transient is considered as two discrete behaviors, with the machine profile parameters being measures of the behaviors both individually (stability) and as a whole (magnitude of change). $|S|$ is inferred through the magnitude between the two behavior centroids, which is determined using simple $k$-means clustering (with $k=2$ ), while the $S_{\text {Lead }}$ and $S_{\text {Tail }}$ tolerances follow the same process as the envelope-based parameters.

\section{B. Selection of data}

The selection of tuning data for both classes of parameters is made through a number of assumptions regarding the operation of the machinery and statistical characteristics of the resultant distributions during the period in question.

1) Envelope-based parameters: The tuning behavior for envelope-based parameters should be selected as the most recent example of steady state, operational behavior. That is, the period of data used as the benchmark should represent normality for the machine at that given point of operation, without any instance of alarm, routine or otherwise.

For the tuning algorithm to identify and reason about the selected behavior correctly, the data must be considered unimodal. Unimodal classification is made through examination of the behavior's $C D F$. Where the $C D F(x)$ is convex < $m o(x)$ and concave $>m o(x)$, it can be said the distribution meets unmorality requirements [23]. With respect to real data, the empirical cumulative density function (ECDF) is determined, and compared against a corresponding unit Gaussian $\mathrm{CDF}$ of $\sigma=1$ with the same mode value. Kolmogorov and Smirnov [24] demonstrated the application of comparison between the empirical and hypothetical CDFs as a measure of the similarity between an empirical and theoretical behavior.

Fig. 5 illustrates both steady state and transient examples compared to a hypothetical CDF with $s$-normal properties. Note that, in the steady state case, the ECDF deviates less from the corresponding ideal Gaussian CDF. From this analysis, the selection of tuning data is based on the similarity between the empirical and hypothetical distributions, with particular focus on the convexity and concavity before and after the distribution mode. Hartigan's dip test [23] measures the maximum deviation $D_{n}(x)$ between the empirical and hypothetical CDFs, providing a statistical metric of similarity between test data and an ideal unimodal function.

2) Instance-based parameters: Selection of suitable instances of step changes is dependent on their identification as routine transients. Tuning to non-routine examples, or alarms corresponding to permanent machine change or damage, would parameterize the machine profile away from normality towards particular fault data. With this effect in mind, the process at this stage of the research is to train on manually selected instances of previous step instances.

Fundamentally, the training examples for this approach are required to correspond to the criteria used within the knowledge-based system in classifying non-fault data deviations. For example, the selection of training examples should be made to best tune $|S|, S_{\text {Lead }}$, and $S_{\text {Tail }}$ to routine step characteristics. $|S|$ is essentially the minimum magnitude recognised by the system as a step change in an observable. Therefore, selected step value tuning data populations should account for the lower extremes of detectable step behavior to set this value. Alongside, the stability of pre-step $S_{\text {Lead }}$ and post-step $S_{\text {Tail }}$ behaviors are required to be considered, as 

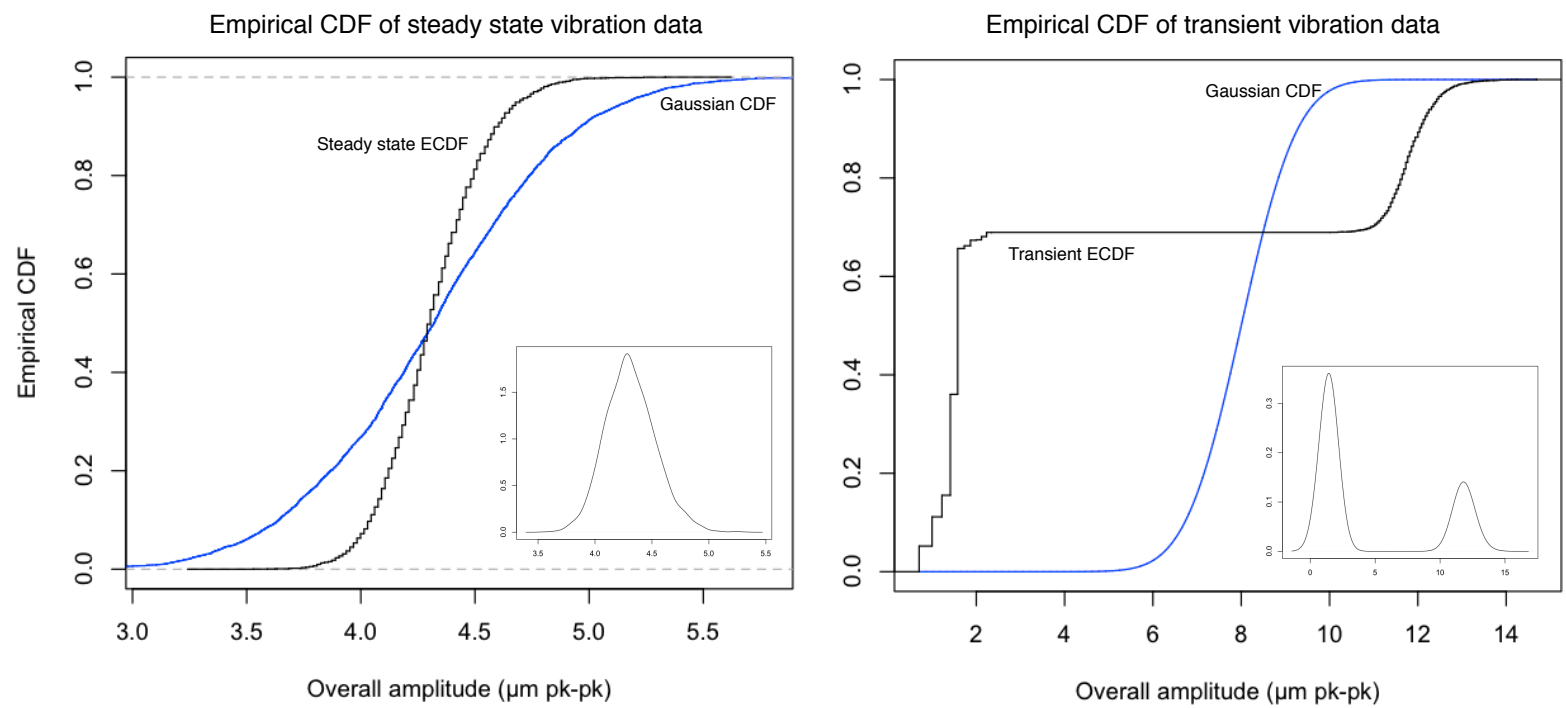

Fig. 5. Examination of the ECDF for both steady state and transient vibration data behaviors.

noisy data prior to or directly following a step change alters the diagnostic conclusion of the system.

The selection of these bounds is made at this stage through knowledge-directed selection corresponding with historical case studies in the area. In selecting tuning data, each of these parameters need to be considered with respect to how normality and benign transients manifest themselves in each of the vibration and operational observables. A major future research area will investigate the automation of this tuning data identification and selection process, keeping close focus on the knowledge and validation issues surrounding the definition of system normality.

\section{Tuning process}

From an asset reliability perspective, it is useful to now discuss how the tuning functionality of the knowledge base operates within the engineering scenario. This discussion illustrates how the diagnosis procedure would be utilized by the vibration monitoring engineer or expert.

Selection of suitable envelope data can be made either manually by the engineer, or identified automatically through steady-state detection techniques. For manual selection, the engineer identifies periods of data corresponding to steady online operation from recent historical records to determine the best representation of normality for the machine at that time. In automatic detection, this selection can be achieved through the use of a windowed Hartigan's dip test, providing a statistical metric for the automatic selection of unimodal steady-state data. Various considerations are required when identifying suitable data in the context of rotating plant operation, such as correlation with duty cycle progress and event history. In the later case study section, both approaches to selecting tuning data are used complimentarily to demonstrate the tuning concept.

Instance-based data are to be selected from previous routine alarm instances of the machine, requiring deeper analysis than the process corresponding to the envelope data. From these selected event instances, the parameters of normality can be extracted from the data through the techniques discussed.

The tuning process then utilizes these learned observable parameters to provide suitable diagnosis on later instances of routine transients. In application, the number of observables would be larger, depending on the rotating asset in question. Fundamentally, however, the algorithm of detecting and correlating features in operational observables to vibration change remains the same.

\section{CASE STUDY}

Throughout this section, an example of real data taken from a turbine generator is used to illustrate the principles discussed in Section II. For clarity, analysis is made of a single vibration observable (overall amplitude) against a single operational observable (generator load). It should be re-iterated, however, that the developed system itself will analyse a multitude of both vibration and operational observables in practice, for each alarm instance, to find the correct routine alarm diagnostic conclusion.

Without the use of the tuning methodology discussed in this paper, the knowledge-based system can only utilize manuallyset parameters for the extraction of the time series primitives. Values for these parameters were originally set heuristically by domain experts, and the current procedure is to use a single generalized machine profile for any turbine generator machine instance. The values of these parameters are derived from early case studies for the knowledge-based system design, loosely corresponding to the International Standards Organization (ISO)-standard [25] vibration alarm limits. Analysis through this section should illustrate that this approach is insufficient to deliver correct routine alarm diagnostics across multiple instances of turbine, which have the potential to exhibit differing characteristics dependent on numerous operational and machine state factors. 


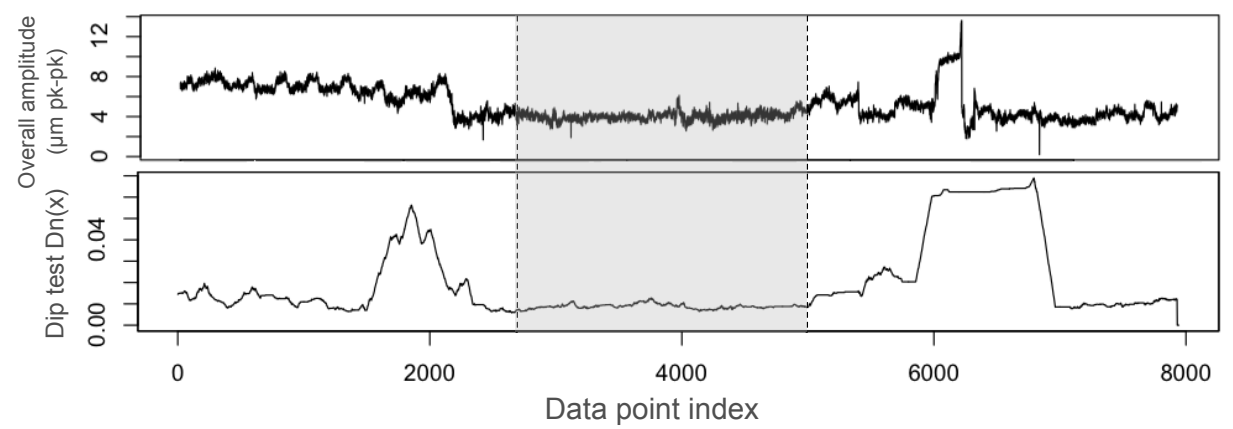

Fig. 7. Utilization of Hartigan's dip test as a metric for unimodality and selection of data based on statistical inference.

\section{A. Envelope free parameters $A$ and $B$}

To correctly identify time series primitives from future alarm data, the free parameters of $A$ and $B$ are required to be selected for the domain application. These settings should be chosen to best represent a knowledge-based view of steady state normality while remaining sensitive to any observable changes from trends or impulses. The correct diagnosis of routine transients is dependent, statistically and otherwise, on how well these free parameters model the primitive time series behaviors. To achieve this end, the procedure is to select values a priori for $A$ and $B$, corresponding to both knowledge-driven and historical information regarding the diagnosis process.

Fig. 8 shows the density functions of historical steady state data populations alongside corresponding alarm deviations as a result of ten routine alarm: five trend-based instances, and five large change, impulse instances. The two alarm types form two distinct groups by deviation magnitude, disregarding temporal effects. To best represent these behaviors, the selected $A$ and $B$ values need to classify these groups successfully while encompassing the steady state data. Presented with these data, the parameters for later analysis were set at $A=1$ and $B=3$, providing ample envelopes for each of the behaviors. The $A$ parameter in particular represents a key value of consideration, as the determination between steady-state and trend behavior is particularly important for the identification of alarm emergence. Deviation of this $A$ value results in misclassification of trend symbols, leading to often incorrect alarm conclusions. These values also corresponded closely with the knowledge-driven elicited values of case studies from earlier in the development of the system. The process described and illustrated in Fig. 8 is suggested to be application dependent, and the values should be informed by both historical examples and tacit knowledge surrounding the diagnostic problem.

\section{B. Data selection and diagnosis}

The alarm for the automated diagnosis process was selected from an operational turbine generator which experienced a routine transient in load, corresponding to short-term system change in station-side operation. Vibration changes for load transients are expected to be replicated across each of the turbine components. This example focuses on the vibration characteristics of the front bearing from the HP stage of the

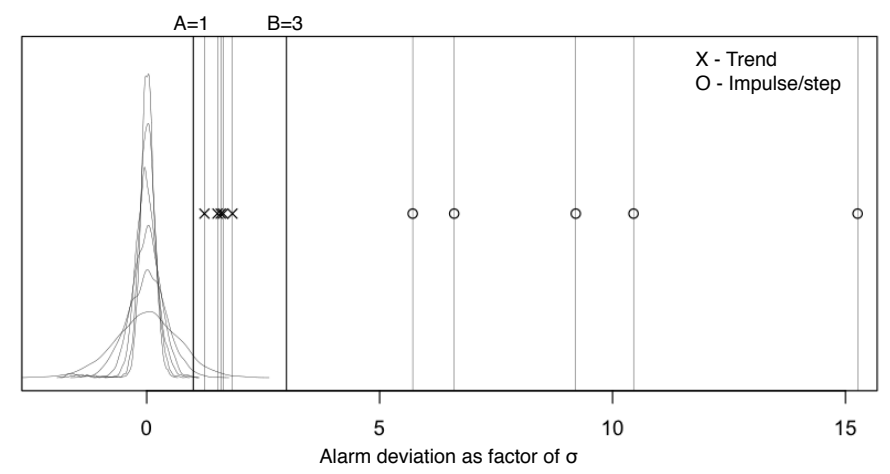

Fig. 8. Historical examples of trend, and large change routine alarm deviation in terms of $\sigma$ values.

turbine. This example is used with corresponding tuning data selected by both manual and automatic means to validate the process.

The previously elicited parameters are denoted in Table II, along with three potential machine profiles tuned by the system. Two utilize manually selected tuning data corresponding to user-identified periods of steady state behavior, while a third uses data selected using the statistical dip test metric from a long-term operational history period. The difference in envelope values is notable between the tuned and untuned parameters, illustrating the machine-specific nature of these machine profile examples in both the manually and automatically selected tuning cases.

TABLE II

COMPARISON OF MANUALLY SET AND TUNED MACHINE PROFILE PARAMETERS ON TURBINE GENERATOR BEARING.

\begin{tabular}{|c|c|ccc|}
\hline Parameter & Manually-set & Sel. tuned $i$ & Sel. tuned $i i$ & Auto-tuned \\
\hline$m o(x)$ & 3.00 & 4.34 & 4.30 & 4.30 \\
$l l(x)$ & 1.75 & 3.35 & 3.63 & 3.62 \\
$l b(x)$ & 2.50 & 4.01 & 4.08 & 4.07 \\
$u b(x)$ & 3.50 & 4.67 & 4.53 & 4.54 \\
$u l(x)$ & 4.25 & 5.33 & 4.98 & 4.99 \\
\hline
\end{tabular}

The discrepancies between these values can be explained by a number of reasons. The knowledge base utilized in the earlier versions of the system used a small subset of routine alarm case studies on a particular asset to provide proof of diagnosis for routine alarm instances. Therefore, with limited experience 
of the parametric values, it can be reasonably assumed that these initialized parameters are non-optimal over all possible cases of routine alarm. More important, however, is the fact that individual turbine generators will exhibit differing characteristics dependent on a variety of factors (orientation, maintenance history, duty cycle, degradation).

The selected data in the windowed dip test example is illustrated in Fig. 7, showing the data itself alongside the statistical metric below. The period in question was taken in the month preceding the alarm instance, with the algorithm identifying the suitable tuning period. The system identifies local minima of the windowed $D_{n}(x)$ value, and selects the largest period of data points with minimal variance in this value. Selection of these data is made through the criteria of dip parameter size and stability, where it is postulated that the 'most unimodal' periods of operation will occur when the difference between the empirical and theoretical CDFs is minimal and steady-state itself. It should be iterated that this technique is in the early phases of use, and numerous issues remain regarding the automatic selection of data vs. validation of the data as suitable for learning. This technique is intended to show the potential for data-driven systems in this realm using statistical inference metrics to help inform intelligent decision making.

Instance-based tuning was made on a number of routine step changes in the operational months preceding the alarm instance. However, the difference between the minimum $|S|$ and stability $S_{\text {Lead }}, S_{\text {Tail }}$ values used in the knowledgebased standard machine profile, and the values taken from newer instances were all found to have negligible difference. The intention for instance-based tuning is broadly to track machine-specific changes, requiring a more detailed long-term analysis of all routine transient instances on a given component channel. It is hoped that such analyses will comprise the main focus of a later study, in search of intra-machine change of operational event response as a potential metric for system degradation.

For testing the diagnosis of the routine alarm, the alarm was examined using all three envelope parameter results for completeness. Due to their relative similarity, the system provided the same final diagnosis each time: a change in vibration behavior caused by a step in generator load. Fig. 9 provides the buffer alarm data of the system, showing the detection of the routine transient after the tuning procedure, along with the symbols fired in diagnosing this alarm in Table III.

TABLE III

GENERATED SYMBOLS FROM THE ALARM EXAMPLE IN FIG. 9 PROVIDING THE LOAD CHANGE DIAGNOSIS.

Vibration (overall amplitude) trend change = FALLING
Vibration (overall amplitude) trend period $>$ Alarm period
Vibration (overall amplitude) change post-alarm = true
Vibration (overall amplitude) trend level $\neq$ LOW
Vibration (overall amplitude) trend level $\neq$ HIGH
Operational (generator load) trend level = FALLING
Operational (generator load) trend period $<$ Alarm period
Operational (generator load) change pre-alarm = true
DIAGNOSIS: Load change cause alarm

Vibration (overall amplitude) trend change $=$ FALLING

Vibration (overall amplitude) trend period $>$ Alarm period

Vibration (overall amplitude) trend level $\neq$ LOW

Vibration (overall amplitude) trend level $\neq \mathrm{HIGH}$

Operational (generator load) trend level = FALLING

DIAGNOSIS: Load change cause alarm
With reference to Table II, see that use of the manually set envelope parameters provides misdiagnosis of the alarm instance due to the difference in the feature extraction parameters. A large proportion of the steady-state behavior being exhibited is above the manually set $u l(x)=4.25$ value. While this result could be altered on an example-by-example basis, in practice the parameters would need to be updated for each alarm instance across a wide number of machine observables. This adjustment would render the procedure particularly onerous, and potentially leave the technique itself (which is being carried out to reduce the diagnosis time of routine transients) more involved than manual engineering analysis.

\section{FUTURE WORK}

The inductive nature of the techniques discussed, and the relative simplicity of the routine alarm rules used, suggests the application of this framework is not limited to steam turbine vibration analysis. Investigations are ongoing in the application of the discussed algorithms to the vibration characteristics of gas circulator units, which represent another major rotating asset in the nuclear generation scenario.

With the parametric framework for defining system normality in place, numerous machine learning techniques are to be investigated to add further autonomy to the data selection step of the tuning process. The steady-state detection technique using Hartigan's dip test remains in the early prototype stage, and requires further experimentation with operational events and duty cycles of greater complexity.

Instance-based tuning data needs to be subject to a longterm turbine investigation, ascertaining the typical evolution of these parameters beyond multiple tuning regimes. A combination of unsupervised learning of time series primitives and reasoning about machine state would allow for the system to itself suggest potential instance tuning data during operation for engineering validation. This automated utilization of unlabeled vibration and operational data would reduce any requirements for manual selection of suitable tuning data examples, instead validating steady state behaviors and routine transients against the statistical techniques discussed earlier in the paper. A system with the ability to accurately determine routine behavior would have the potential to continually tune and re-tune automatically following major transients, outages, and maintenance cycles. This re-tuning would allow for the evolution of the machine profile with operation to be accounted for, following any change of state with the asset.

Further to this approach, it is postulated that a self-tuning algorithm with the ability to accurately follow long-term changes in system state and update the machine profile accordingly will have notable potential for prognostic analysis. With deeper understanding of the parametric machine view, and any potential system metrics taken from future analyses, predictive calculations are possible to be made to inform of expected future behaviors of the asset in question. However, as with many of the early attempts into the application of prognostic techniques to data-rich engineering problems [7], [26], the mapping of a degradation function and sensitivity to deviations in behavior presents the major research problem to be tackled. 


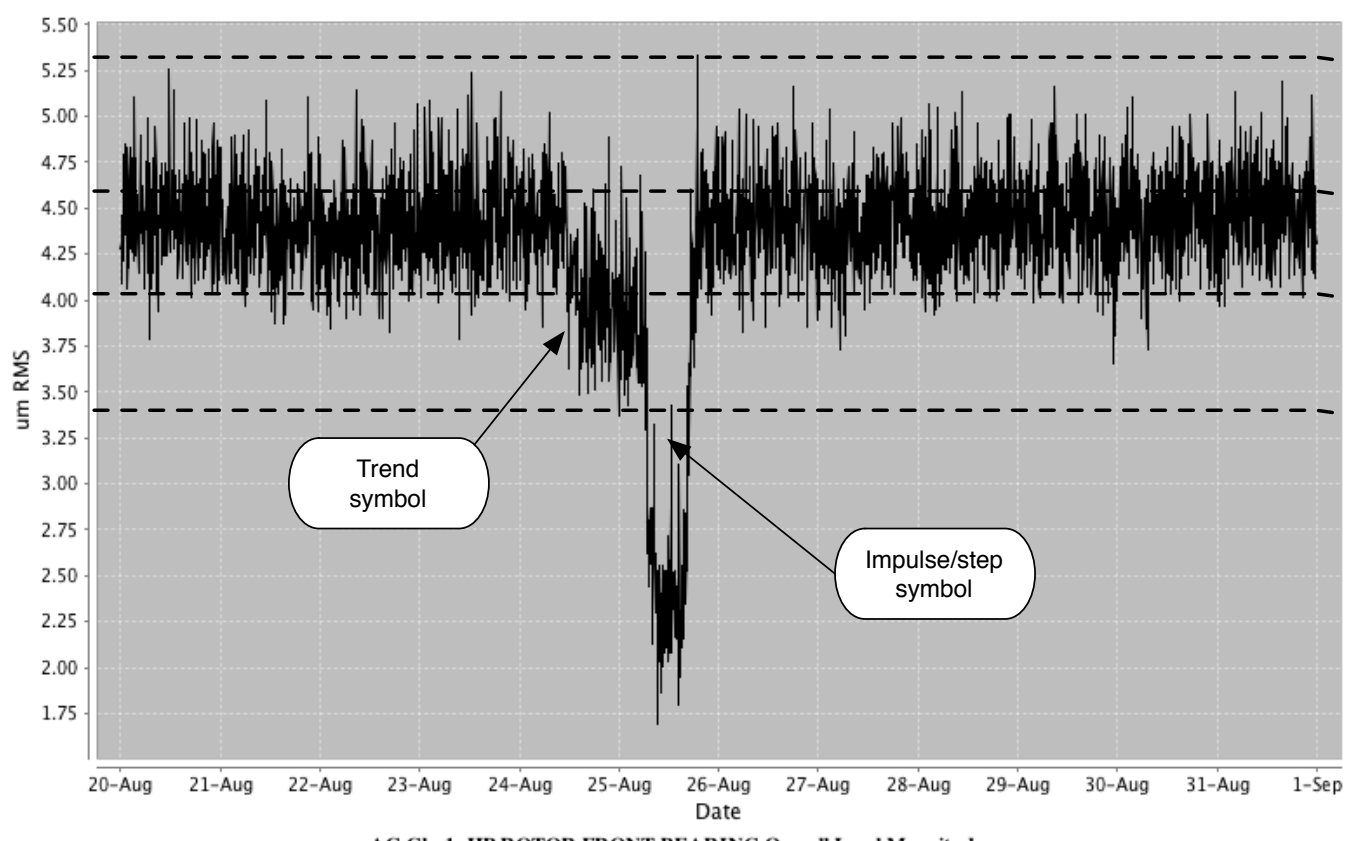

Fig. 9. Alarm identification and classification by the system, highlighting the deviation from normal behavior.

\section{CONClusions}

This paper has outlined a framework for the automated diagnosis of routine vibration alarm instances utilizing selftuning techniques, allowing for decision support to be provided on multiple instances of steam turbine generators. A combination of knowledge-based heuristics and statistical inference provides a system with high adaptability to individual machine characteristics. This approach allows for the accurate diagnosis of routine alarm instances across multiple instances of steam turbines. This work is particularly advantageous to any asset management team with multiple machines under their scrutiny.

The approaches discussed are anticipated to provide the foundation for further investigation into self-tuning diagnosis and long-term analysis, where the individual characteristics of each asset continue to be considered and reasoned about through the use of statistical and intelligent system techniques.

\section{ACKNOWLEDGEMENTS}

This work was funded by EDF Energy and EPSRC. The views presented by the authors do not necessarily represent the views of EDF Energy.

\section{REFERENCES}

[1] R. B. Randall, "State of the Art in Monitoring Rotating Machinery Part 1," Sound and Vibration, pp. 14-20, 2004.

[2] P. J. Tavner, "Review of condition monitoring of rotating electrical machine," IET Electric Power Applications, vol. 2, no. 4, pp. 215-247, 2008.

[3] D. Jiang and C. Liu, "Machine Condition Classification Using Deterioration Feature Extraction and Anomaly Determination," IEEE Transactions on Reliability, vol. 60, no. 1, pp. 78-88, 2011.

[4] E. Zio and G. Gola, "A neuro-fuzzy technique for fault diagnosis and its application to rotating machinery," Reliability Engineering \& System Safety, vol. 94, no. 1, pp. 7888, Jan. 2009. [Online]. Available: http://linkinghub.elsevier.com/retrieve/pii/S095183200700138X
[5] L. Trave-Massuyes and R. Milne,"Gas-turbine condition monitoring using qualitative model-based diagnosis," IEEE Expert, vol. 12, no. 3, pp. 2231, May 1997. [Online]. Available: http://ieeexplore.ieee.org/lpdocs/epic03/wrapper.htm?arnumber=590070

[6] B. Yang, D. Lim, and A. Tan,"VIBEX: an expert system for vibration fault diagnosis of rotating machinery using decision tree and decision table," Expert Systems with Applications, vol. 28, no. 4, pp. 735742, May 2005. [Online]. Available: http://linkinghub.elsevier.com/retrieve/pii/S0957417404001770

[7] A. Heng, S. Zhang, A. Tan, and J. Mathew, "Rotating machinery prognostics: State of the art, challenges and opportunities," Mechanical Systems and Signal Processing, vol. 23, no. 3, pp. 724739, Apr. 2009. [Online]. Available: http://linkinghub.elsevier.com/retrieve/pii/S0888327008001489

[8] M. Todd, S. D. J. McArthur, J. R. McDonald, and S. J. Shaw, "A Semiautomatic Approach to Deriving Turbine Generator Diagnostic Knowledge," IEEE Transactions on Systems, Man, and Cybernetics, Part C: Applications and Reviews, vol. 37, no. 5, pp. 979992, 2007.

[9] J. J. A. Costello, G. M. West, S. D. J. McArthur, and G. Campbell, "Self-tuning diagnosis of routine alarms in rotating plant items," in The Eighth International Conference on Condition Monitoring and Machinery Failure Prevention Technologies, 2011.

[10] A. Jardine, D. Lin, and D. Banjevic, "A review on machinery diagnostics and prognostics implementing condition- based maintenance," Mechanical Systems and Signal Processing, pp. 14831510, Oct. 2006. [Online]. Available: http://linkinghub.elsevier.com/retrieve/pii/S0888327005001512

[11] W. C. Flores, E. E. Mombello, J. a. Jardini, G. Ratta ?, and A. M. Corvo, Expert system for the assessment of power transformer insulation condition based on type- 2 fuzzy logic systems, Expert Systems with Applications, vol. 38, no. 7, pp. 81198127, Jul. 2011. [Online]. Available: http://linkinghub.elsevier.com/retrieve/pii/S0957417410015162

[12] S. Rudd, S. D. J. McArthur and M. Judd,"A generic knowledgebased approach to the analysis of partial discharge data," IEEE Transactions on Dielectrics and Electrical Insulation, vol. 17, no. 1, pp. 149156, Feb. 2010. [Online]. Available: http://ieeexplore.ieee.org/lpdocs/epic03/wrapper.htm?arnumber=5412013

[13] E. Davidson, S. D. J. McArthur, J. McDonald, T. Cumming, and I. Watt, Applying Multi-Agent System Technology in Practice: Automated Management and Analysis of SCADA and Digital Fault Recorder Data, IEEE Transactions on Power Systems, vol. 21, no. 2, pp. 559567, May 2006. [Online]. Available: http://ieeexplore.ieee.org/lpdocs/epic03/wrapper.htm?arnumber=1626359

[14] T. Mitoma, H. Wang, and P. Chen, "Fault diagnosis and con- 
dition surveillance for plant rotating machinery using partially linearized neural network," Computers \& Industrial Engineering, vol. 55, no. 4, pp. 783794, Nov. 2008. [Online]. Available: http://linkinghub.elsevier.com/retrieve/pii/S0360835208000570

[15] V. A. Sotiris, P. W. Tse, and M. G. Pecht, Anomaly detection through a Bayesian Support Vector Machine, IEEE Transactions on Reliability, vol. 59, no. 2, pp. 277286, 2010.

[16] D. A. Clifton, M. S. Park, L. Tarassenko, N. McGrogan, D. King, S. King, P. Anuzis, and R.-r. Plc, Bayesian Extreme Value Statistics for Novelty Detection in Gas-Turbine Engines, in IEEE Aerospace Conference, pp. 111, 2008.

[17] E. Zio, P. Baraldi, and G. Gola, Feature-based classifier ensembles for diagnosing multiple faults in rotating machinery, Applied Soft Computing, vol. 8, no. 4, pp. 13651380, Sep. 2008. [Online]. Available: http://linkinghub.elsevier.com/retrieve/pii/S1568494607001287

[18] D. Simon and D. L. Simon, Analytic Confusion Matrix Bounds for Fault Detection and Isolation Using a Sum-of-Squared-Residuals Approach, IEEE Transactions on Reliability, vol. 59, no. 2, pp. 287 296, 2010.

[19] Z. Hameed, Y. Hong, Y. Cho, S. Ahn, and C. Song, Condition monitoring and fault detection of wind turbines and related algorithms: A review, Renewable and Sustainable Energy Reviews, vol. 13, no. 1, pp. 139, Jan. 2009. [Online]. Available: http://linkinghub.elsevier.com/retrieve/pii/S1364032107001098

[20] Y. K. Lee, D. N. Mavris, V. V. Volovoi, M. Yuan, and T. Fisher, A Fault Diagnosis Method for Industrial Gas Turbines Using Bayesian Data Analysis, Journal of Engineering for Gas Turbines and Power, vol. 132, no. 4, p. 041602, 2010.

[21] G. Schreiber, H. Akkermans, A. Anjewierden, R. de Hoog, N. Shadbolt, W. Van De Velde, and B. Wielinga, Knowledge Engineering and Management, 2000

[22] D. G. Galati and M. A. Simaan, Automatic decomposition of time series into step, ramp, and impulse primitives, Pattern Recognition, vol. 39, no. 11, pp. 21662174, Nov. 2006. [Online]. Available: http://linkinghub.elsevier.com/retrieve/pii/S0031320305003833

[23] J. A. Hartigan and P. M. Hartigan, The Dip Test of Unimodality, The Annals of Statistics, vol. 13, no. 1, pp. 7084, 1985.

[24] F. J. Massey, The Kolmogorov-Smirnov test for goodness of fit, American Statistical Association, vol. 46, no. 253, pp. 68 78, 1951.

[25] ISO 22266-1:20009: Mechanical vibration - Torsional vibration of rotating machinery - Part 1: Land-based steam and gas turbine generator sets in excess of 50MW, International Organization for Standardization, 2009.

[26] D. B. Jarrell, D. R. Sisk, and L. J. Bond, Prognostics and ConditionBased Maintenace: A New Approach to Precursive Metrics, Nuclear Technology, 2003.
Jason J. A. Costello received the BSc. (Hons) in Applied Physics in 2009 from the University of Strathclyde. He is currently undertaking an Eng.D at the Institute of Energy \& Environment in the Department of Electronic and Electrical Engineering at the University of Strathclyde. His research interests include AI applications in condition monitoring, dynamics of rotating plant items, and machine learning.

Graeme M. West is a research fellow at the Institute for Energy and Environment in the Department of Electronic and Electrical Engineering at the University of Strathclyde. He received a BEng (Hons) degree in Electrical and Mechanical Engineering, and a PhD in Electrical Engineering in 1998, and in 2002 respectively, both from the University of Strathclyde. His current research interests include intelligent system applications and data mining within power engineering, and in particular applying the techniques to applications within the nuclear industry.

Stephen D. J. McArthur (M'93-SM'07) received the B.Eng. (Hons.), and Ph.D. degrees from the University of Strathclyde, Glasgow, U.K., in 1992, and 1996, respectively. He is co-Director of the Institute for Energy and Environment, and Director of the EDF Energy Advanced Diagnostics Centre at the University of Strathclyde 